\title{
Pineal region hemangioblastoma in a patient with Von Hippel-Lindau disease
}

\author{
Gustavo Rassier Isolan 1,2, Patrícia Ashton-Prolla, \\ Marcelo Martins dos Reis, Ápio Cláudio Martins Antunes
}

To the best of our knowledge there are only three previous reports of hemangioblastoma localized in the pineal region. Two in patients with Von Hippel-Lindau (VHL) disease ${ }^{1}$ and one in patient without VHL disease ${ }^{2}$.

\section{CASE}

The patient is a 37 year-old female who was diagnosed with Von Hippel-Lindau disease at the age of 28 years. At presentation the patient had an abdominal sonogram showing pancreatic cysts and a single liver hemangioma, normal ophthalmologic examination and normal CT scan of the brain. One year later, she had an episode of vertigo and severe headache and a new CT scan of the brain showed an space-ocupying lesion in the left cerebellar hemisphere compatible with hemangioblastoma. Surgical resection was performed. Mutation screening of the VHL gene was performed and a frameshift mutation in exon two (codon 115) was identified (g.344delA).

Follow-up studies showed an expansive mass of the pancreatic head. She developed persistent headache and a MRI of the brain showed a giant brightly mass in the quadrigeminal cistern causing hydrocephalus. The physical examination did not show any focal deficit. Fundoscopy showed bilateral papilledema. The tumor was ressected. Postoperative hydrocephalus developed and was treated with ventriculoperitoneal shunt. The microscopic features showed a hemangioblastoma. The symptoms of intracranial hypertension improved.

Three months later, the patient clinical status deteriorated. A new MRI revealed a large posterior fossa cyst with a large mural nodule at the topography of the posterior incisural space. The tumor was completely ressected en bloc. The anterior wall of the cyst was open to stablish a communication with the fourth ventricule. The pathology was hemangioblastoma. The patient symptoms improved. The tumor was immunopositive for neuronspecific enolase (NSE) and immunonegative for epithelial membranous antigen (EMA).

\section{DISCUSSION}

Von Hippel-Lindau syndrome is an autosomal dominant cancer predisposition syndrome caused by germline mutations in the VHL gene, a tumor suppressor gene localized at chromosome 3p25-26. Renal cell carcinoma occurs in about $40 \%$ of individuals with VHL and is the leading cause of mortality. The clinical criteria for VHL disease are those from Melmon and Rosen ${ }^{3}$. The analysis for the presence of VHL gene mutation can be done with a peripheral blood test.

The differential diagnosis between CNS hemangioblastoma and metastatic clear cell renal cell carcinoma can be difficult. Immunohistochemical studies can be helpful in this setting: hemangioblastomas do not have an epithelial origin and so do not express EMA, while renal cell carcinomas show immunopositivity for EMA and high percentage of CD10 staining. Furthermore, hemangioblastomas typically display immunopositivity for NSE and inhibin A, unlike renal cell carcinomas ${ }^{4,5}$.

Isolan et al. reported a case of a pineal region hemangioblastoma causing hydrocephalus ${ }^{2}$. The tumor was adherent to the quadrigeminal plate and the patient evolved well after tumoral resection en bloc. The criteria to VHL disease were absent. Based on this first case, we prefer to resect the tumor en bloc instead of trying to resect piece by piece.

This unusual association highlights the importance of considering this lesion in the differential diagnosis of pineal region masses.

\section{REFERENCES}

1. Sajadi A, de Tribolet N. Unusual locations of hemangioblastomas: case illustration. J Neurosurg 2002;97:727.

2. Isolan GR, Krayenbühl N, Mahmoud M, Al-Mefty O. A hemangioblastoma in the pineal region: case report. Neurosurgery 2007;61:423.

3. Melmon KL, Rosen SW. Lindau's disease: review of the literature and study of large kindred. Am J Med 1964; 36:595-617.

4. Gouldesbrough DR, Bell JE, Gordon A. Use of immunohistochemical methods in the differential diagnosis between primary cerebellar haemangioblastoma and metastatic renal cell carcinoma. J Clin Pathol 1988; 41:861-865

5. Hoang MP, Amirkhan RH. Inhibin alpha distinguishes hemangioblastoma from clear cell renal cell carcinoma. Am J Surg Pathol 2003;27:1152-1156.

HEMANGIOBLASTOMA DA REGIÃO PINEAL EM PACIENTE COM DOENÇA DE VON HIPPEL-LINDAU

${ }^{1}$ Department of Neurology and Neurosurgery, Hospital de Clínicas de Porto Alegre (HCPA), Porto Alegre RS, Brazil; ${ }^{2}$ Medicine Postgraduation Program in Surgery, HCPA; ${ }^{3}$ Department of Genetics, HCPA.

Correspondence: Gustavo Rassier Isolan - Serviço de Neurologia - Rua Ramiro Barcelos 2350 - 90035-903 Porto Alegre RS - Brasil. E-mail: gisolan@yahoo.com.br Received 17 May 2011. Received in final form 6 June 2011. Accepted 13 June 2011. 